\title{
Solar neutrino results and Violation of the Equivalence Principle: An analysis of the existing data and predictions for SNO
}

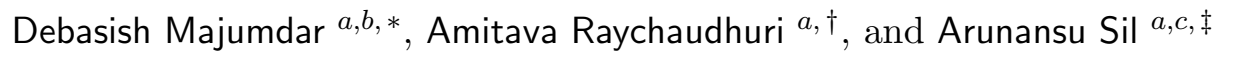 \\ ${ }^{a}$ Department of Physics, University of Calcutta, 92 Acharya Prafulla Chandra Road, \\ Calcutta 700009, India. \\ ${ }^{b}$ Saha Institute of Nuclear Physics, \\ 1/AF Bidhannagar, Calcutta 700064, India. \\ ${ }^{c}$ Department of Physics, Jadavpur University, Calcutta 700032, India.
}

\begin{abstract}
Violation of the Equivalence Principle (VEP) can lead to neutrino oscillation through the nondiagonal coupling of neutrino flavor eigenstates with the gravitational field. The neutrino energy dependence of this oscillation probability is different from that of the usual mass-mixing neutrino oscillations. In this work we explore, in detail, the viability of the VEP hypothesis as a solution to the solar neutrino problem in a two generation scenario with both the active and sterile neutrino alternatives, choosing these states to be massless. To obtain the best-fit values of the oscillation parameters we perform a $\chi^{2}$ analysis for the total rates of solar neutrinos seen at the Chlorine (Homestake), Gallium (Gallex and SAGE), Kamiokande, and SuperKamiokande (SK) experiments. We find that the goodness of these fits is never satisfactory. It markedly improves, especially for VEP transformation to sterile neutrinos, if the Chlorine result is excluded from the analysis. The 1117-day SK data for recoil electron spectrum are also examined for signals of VEP oscillations. For these fits, ' we consider variations of the Standard Solar Model by allowing the absolute normalizations of the ' ${ }^{8} \mathrm{~B}$ and hep neutrinos to vary. Here the fits are quite good but the best fit values of the parameters are rather different from those from the total rates fits. A combined fit to the total rates and recoil electron spectrum data is also performed. We present the $90 \%$ confidence limit contours for all the three analyses mentioned above. The best-fit parameters obtained from the recoil electron spectrum and the combined analysis of rate and spectrum are used to predict the charge current and scattering electron spectrum at SNO.
\end{abstract}

PACS NO. 26.65.+t, 14.60.Lm, 14.60.Pq, 04.80.Cc

\footnotetext{
${ }^{*}$ E-mail address: debasish@tnp.saha.ernet.in

${ }^{\dagger}$ E-mail address: amitava@cubmb.ernet.in

${ }^{\ddagger}$ E-mail address: arun@cubmb.ernet.in
} 


\section{Introduction}

Oscillation of neutrinos from one flavor to another is currently the favored solution to the solar neutrino problem [1], 2, 3]. This proposition has been strengthened by the recent SuperKamiokande (SK) atmospheric neutrino data [4] which also support the existence of neutrino oscillations. The usual formulation of neutrino oscillations rests on two essential properties; namely, (a) the neutrinos are massive and are further not mass degenerate, and (b) the flavor eigenstates (i.e., $\nu_{e}, \nu_{\mu}$, and $\nu_{\tau}$ ) are not themselves the eigenstates of mass but rather linear superpositions of the latter. Distinct from the simplest vacuum oscillations, neutrino flavor conversion can also be induced by the passage of neutrinos in matter (in this case solar matter) due to the difference in the strength of weak interactions of neutrinos of different flavor with ambient electrons. This is the Mikheyev-Smirnov-Wolfenstein (MSW) [5] effect. Vacuum oscillations and the MSW effect are widely considerd to be strong candidates for the solution of the solar neutrino problem. Much work has already been done to analyze the available solar neutrino data in terms of these alternate possibilities and substantial effort is still being devoted to obtain the parameters that best fit the data [6, 7, 8, 9, 10, 11].

Though the vacuum oscillation and MSW solutions relying on massive neutrinos (we refer to these collectively as 'mass-mixing' solutions henceforth) are by far the most popular scenarios for addressing the solar and atmospheric neutrino data, oscillations can also originate from other sources. One such possibility presents itself if violation of the weak equivalence principle (VEP) occurs and the flavor eigenstates are not identical to the states that couple to gravity. The principle of equivalence is a cornerstone of Einstein's general theory of relativity. Normally such a premise would be considered sacrosanct, but so little has been experimentally tested for neutrinos that it may not be unreasonable to keep an open mind and check the validity of this principle for them. If this principle is indeed violated then, as a consequence, the coupling of neutrinos to the gravitational field is

nonuniversal. Under this circumstance, if the flavor eigenstates are linear superpositions of the gravitational eigenstates, VEP induced oscillations of neutrinos take place [12]. This does not require neutrinos to carry a non-zero mass. The important difference between this approach and the mass-mixing solution is manifested in the energy dependence of the survival probability. For a two-neutrino picture, the general expression for the survival probability for an initial $\nu_{e}$ after propagation through a distance $L$ is given by:

$$
P_{e e}\left(E_{\nu}, L\right)=1-\sin ^{2} 2 \theta \sin ^{2}\left(\frac{\pi L}{\lambda}\right),
$$

where $\theta$ is the rotation angle relating the gravitational eigenstate basis $\left(\nu_{1}, \nu_{2}\right)$ to the flavor basis $\left(\nu_{e}, \nu_{x}, x=\mu, \tau\right.$, or a sterile state).

$$
\nu_{e}=\nu_{1} \cos \theta+\nu_{2} \sin \theta ; \quad \nu_{x}=-\nu_{1} \sin \theta+\nu_{2} \cos \theta
$$

and $\lambda$ is the oscillation length, which for the VEP induced oscillation is:

$$
\lambda=\frac{2 \pi}{E_{\nu} \phi \Delta f}
$$


where $E_{\nu}$ is the neutrino energy, $\phi$ the gravitational potential, and $\Delta f=f_{1}-f_{2}$ is a measure of the violation of equivalence principle, $f_{i}$ being the coupling strength of the gravitational eigenstates. In contrast, for mass-mixing vacuum oscillations $\lambda=\left(4 \pi E_{\nu}\right) / \Delta m^{2}$, where $\Delta m^{2}$ is the mass square difference between two neutrino species. Thus, for the latter case $\lambda \propto E_{\nu}$ while for VEP $\lambda \propto 1 / E_{\nu}$. Due to the different energy dependences of the survival probability in the mass-mixing and the VEP alternatives, their predictions can be quite different. The phenomenological consequences of VEP-driven neutrino oscillations has attracted attention over the past decade [13].

A completely unrelated situation which also leads to neutrino oscillations with $\lambda \propto 1 / E_{\nu}$ is a recently proposed picture of violation of special relativity (VSR) [14]. If special relativity is violated, the maximum attainable speed of a particle in vacuo need not universally be the speed of light $c$. In particular, if the maximum possible velocities of two types of neutrinos be $v_{1}$ and $v_{2}$ and these velocity eigenstate neutrinos be related to the $\nu_{e}$ and $\nu_{x}$ through a mixing angle $\theta$ (see Eq.(2)) then the survival probability of a $\nu_{e}$ takes the same form as Eq.(11). In this case the expression for $\lambda$ is:

$$
\lambda=\frac{2 \pi}{E_{\nu} \Delta v}
$$

where $\Delta v$ is the velocity difference for the neutrinos $\nu_{1}$ and $\nu_{2}$. Comparing Eqs. (4) and (3) one finds that the energy dependence of the oscillation length is identical in the two cases and the role of $\Delta v$ in the VSR case is the same as that of $\phi \Delta f$ in the VEP formalism. Here, we use the terminology of the VEP mechanism but the results can be taken over mutatis mutandis to the VSR situation.

In this work we make a detailed examination of the VEP scenario in the light of the solar neutrino data. We consider the two possibilities of oscillation of the electron neutrino to (a) another active neutrino $\left(\nu_{\mu}\right.$ or $\left.\nu_{\tau}\right)$, and (b) to a sterile (i.e., no weak interactions) neutrino. The Chlorine and Gallium experiments use radiochemical neutrino detection techniques and do not distinguish between oscillation of the $\nu_{e}$ to an active neutrino or to a sterile one. The Kamiokande and SuperKamiokande experiments, on the other hand, are sensitive to other active neutrinos via the smaller neutral current contribution. Hence, the latter discriminates between the two alternatives of oscillation to active or sterile neutrinos.

In section II, we present a brief summary of the ingredients that go into our analysis.

In the next section we consider the total rates of solar neutrinos observed by the Chlorine, Gallex, SAGE, Kamiokande, and SuperKamiokande (1117-day data) experiments] [3, 1, 2]. We obtain the best-fit values of the VEP parameters and find that the goodness-of-fit? (g.o.f) is never high. We trace the origin of this to the difficulty within the VEP mechanism of simultaneously satisfying the Chlorine detector results and the very precise measurements of SK. We examine the effect of excluding either the Chlorine or the Kamiokande result in

\footnotetext{
${ }^{1}$ It has been shown that inclusion of CPT-violating interactions in addition to Violation of Special Relativity can lead to more general energy dependences involving $1 / E_{\nu}, E_{\nu}$, and constant terms [15]

${ }^{2}$ In the following, we refer to this data set as the 'total rates'.

${ }^{3}$ The goodness of fit gives the probability for the actual $\chi^{2}$ to exceed $\chi_{\min }^{2}$.
} 
the fits or of using the average of the two Gallium results rather than their individual measurements and find that the best-fit values are markedly different only in one situation, the exclusion of the Chlorine data from the analysis, when the quality of the fit is significantly improved.

In section IV we turn to the 1117-day recoil electron energy spectrum from SK [1] and test the ability of the VEP model to account for the observations. In this case the quality of the fit is rather good. We examine a variant of the Standard Solar Model (SSM) in which the absolute normalization of the ${ }^{8} \mathrm{~B}$ neutrinos $\left(X_{B}\right)$ is allowed to vary. In our analysis we have included the contribution from hep neutrinos and we have also examined the situation when the normalization of this flux $\left(X_{h}\right)$ is different from the SSM value of unity.

Section V deals with the simultaneous fitting of the total rates data and the SK 1117-day electron energy spectrum results. We find that good fits can be obtained, in this case, only if $X_{B}$ and $X_{h}$ are allowed to assume values different from the SSM stipulations.

In section VI we turn to the expectations for SNO. We use the best fit values obtained from the above analyses to check how the predictions for the charged current deuteron disintegration and electron scattering are affected by VEP.

We end in section VII with the conclusions. Similar work analysing solar neutrino data using the VEP formalism have been performed in the past [16, 17] and more recently in [18, 19, 20]. We compare our findings with these results.

\section{Solar neutrinos and VEP}

The sun serves as a good neutrino factory. The fusion reactions that generate solar energy also produce neutrinos which are usually denoted by the different reactions (e.g., pp, Be, $B$, $h e p$, etc.) from which they originate. The shape of the neutrino energy spectrum from any reaction is precisely known from weak interaction theory while the absolute normalization of the spectrum depends on the solar parameters like core temperature, opacity, etc. The $p p$ neutrinos are the most copious but they are also of the lowest energy. Only the Gallium experiments (Gallex and SAGE) are sensitive to $p p$ neutrinos and receive their dominant contributions from this process, in addition to smaller ones from the other reactions. At the other extreme, the Kamiokande and SuperKamiokande experiments are based on the water Cerenkov technique and can detect neutrinos of energy higher than about $5 \mathrm{MeV}$. Only the ${ }^{8} \mathrm{~B}$ neutrinos (and to a small extent the hep neutrinos) contribute at these energies. The Chlorine experiment has a threshold of $0.8 \mathrm{MeV}$ and mainly counts ${ }^{7} \mathrm{Be}$ and ${ }^{8} \mathrm{~B}$ neutrinos. Thus the different detectors have probed different regions of the solar neutrino spectrum. Assuming that oscillations do occur during the passage of the neutrinos from their point of origin to the detector, the dependence of this phenomenon on the neutrino energy can be tested by the complementary information from the different experiments. From these results one should be in a position to check which of the vacuum, MSW, or VEP oscillations is preferred by the results. The SuperKamiokande collaboration has also presented the observed energy spectrum of the electron scattered by the incident neutrino. This spectrum is a direct probe of the energy dependence of neutrino oscillations. 
In this work we use the BP98 calculation [21] of the solar neutrino spectrum with INT normalization as the SSM reference. In addition to this, we explore the possibility of the absolute normalizations of the ${ }^{8} \mathrm{~B}$ - and hep-neutrinos spectra being different from their BP98 SSM predictions. If $X_{B}$ and $X_{h}$ denote the factors by which the absolute normalizations are multiplied, we use the data to find the best-fit values for these. In particular, we find that the fit to the SK recoil electron spectrum data is improved in a noteworthy manner when $X_{h}$ is permitted to assume large valuest?

The weak equivalence principle requires the coupling of particles to the ambient gravitational potential $\phi$ to be uniform, i.e., of the form $f \phi E$, where $E$ is the particle energy, and $f$ a universal coupling constant. If the latter varies from one neutrino species to another then that would constitute a violation of the equivalence principle. If $f_{1} \neq f_{2}$ in a two-neutrino framework, then these states define a basis in the two-dimensional space which, in general, could be different from the flavor basis. The effect due to a small splitting $\Delta f$ will manifest itself in the form of flavor oscillations, the wavelength going to infinity as $\Delta f$ tends to zero. We follow the prevalent practice of choosing the gravitational potential, $\phi$, to be a constant over the neutrino path. This is the case if the potential due to the Great Attractor 22] dominates over that due to the sun and other heavenly bodies in our neighborhood. In such an event, writing $\Delta F=\phi \Delta f / 2$, the expression for the oscillation wavelength, Eq. (3) becomes

$$
\lambda=\frac{\pi}{E_{\nu} \Delta F}=\frac{6.20 \times 10^{-13} \mathrm{~m}}{\Delta F}\left(\frac{1 \mathrm{MeV}}{E_{\nu}}\right) .
$$

We choose the neutrinos to be massless.

\section{Observed rates and VEP oscillation of neutrinos}

The data used for the $\chi^{2}$ analysis of total rates are given in Table 1 . They are from the Chlorine experiment at Homestake, the two Gallium experiments, Gallex and SAGE, the water Cerenkov detector Kamiokande, [3], and the latest 1117-day data from SuperKamiokande [1].

\begin{tabular}{|c|c|c|c|c|c|}
\hline Experiment & Chlorine & \multicolumn{2}{|c|}{ Gallium } & Kamiokande & $\begin{array}{c}\text { Super } \\
\text { Kamiokande }\end{array}$ \\
\cline { 3 - 4 } & & Gallex & SAGE & & $0.465 \pm 0.015$ \\
\hline$\frac{\text { Observed Rate }}{\text { BP98 Prediction }}$ & $0.33 \pm 0.029$ & $0.60 \pm 0.06$ & $0.52 \pm 0.06$ & $0.54 \pm 0.07$ & $0.52 \pm 0$ \\
\hline
\end{tabular}

Table 1: The ratio of the observed solar neutrino rates to the corresponding BP98 SSM predictions used in this analysis. The results are from Refs. [3] and [1].

The definition of $\chi^{2}$ used for this analysis is:

$$
\chi^{2}=\sum_{i, j=1,5}\left(F_{i}^{t h}-F_{i}^{e x p}\right)\left(\sigma_{i j}^{-2}\right)\left(F_{j}^{t h}-F_{j}^{e x p}\right) .
$$

\footnotetext{
${ }^{4}$ See, however, remarks in the penultimate paragraph of section VII on the fits to the separate day and night SK electron spectra measurements.
} 
Here $F_{i}^{\xi}=T_{i}^{\xi} / T_{i}^{B P 98}$ where $\xi$ is th (for the theoretical prediction) or $\exp$ (for the experimental value) and $T_{i}$ is the total rate in the $i$-th experiment. $F_{i}^{\text {exp }}$ is taken from Table 1. The error matrix $\sigma_{i j}$ contains the experimental errors, the theoretical errors and their correlations. The theoretical errors have contributions which originate from uncertainties in the detector cross-sections as well as from astrophysics [23]. The off-diagonal elements in the error matrix come through the latter. In estimating the astrophysical contribution, the uncertainties in the spectrum due to the input parameters are taken from [21].

In the presence of neutrino conversions, the detection rate on earth for the radiochemical Chlorine and Gallium experiments is predicted to be:

$$
T_{i}^{t h}=\sum_{\alpha} \int_{E_{t h}} X_{\alpha} \phi_{\alpha}\left(E_{\nu}\right) \sigma_{i}\left(E_{\nu}\right)<P_{e e}\left(E_{\nu}\right)>_{\alpha} d E_{\nu}
$$

where $\sigma_{i}\left(E_{\nu}\right)$ is the neutrino capture cross-section for the $i$-th detector [24] and $E_{t h}$ the neutrino threshold energy for detection. $\phi_{\alpha}\left(E_{\nu}\right)$ stands for the neutrino spectrum for the $\alpha$-th source [24] and $X_{\alpha}$ is an overall normalization factor for this spectrum such that $X_{\alpha}=1$ corresponds to the SSM. The sum is over all the individual neutrino sources. $\left\langle P_{e e}\left(E_{\nu}\right)\right\rangle_{\alpha}$ is the neutrino survival probability for the $\alpha$-th source averaged over the distribution of neutrino production regions in the sun,

$$
<P_{e e}\left(E_{\nu}\right)>_{\alpha}=\int d r P_{e e}\left(E_{\nu}, r\right) \Phi_{\alpha}(r) .
$$

$\Phi_{\alpha}(r)$ is a normalized function which gives the probability of the $\alpha$-th reaction occuring at a distance $r$ from the center of the sun and $P_{e e}\left(E_{\nu}, r\right)$ is obtained by averaging the survival probability over a year taking the eccenticrity of the earth's orbit into account, i.e.,

$$
P_{e e}\left(E_{\nu}, r\right)=\frac{1}{T} \int_{0}^{T} d t\left[1-\sin ^{2} 2 \theta \sin ^{2}\left\{\frac{\pi R(t)}{\lambda}\left(1-\frac{r}{R(t)}\right)\right\}\right]
$$

where $R(t)$ is the sun-earth distance given by,

$$
R(t)=R_{0}\left[1-\epsilon \cos \left(2 \pi \frac{t}{T}\right)\right] .
$$

Here, $R_{0}=1.49 \times 10^{11} \mathrm{~m}$ is the mean Sun-Earth distance and $\epsilon=0.0167$ is the ellipticity of the earth's orbit. $t$ is the time of the year at which the solar neutrino flux is measured and $T$ is 1 year.

The theoretical prediction according to the BP98 Standard Solar Model, $T_{i}^{B P 98}$, is obtained by setting the survival probability as 1 in the above.

For the water Cerenkov detectors Kamiokande and SuperKamiokande, in the case of oscillation to active neutrinos one has to take into account the contributions to the signal from the produced $\nu_{\mu}$ or $\nu_{\tau}$ via the neutral current interactions,

$$
\begin{aligned}
T_{K, S K}^{t h}= & \sum_{\alpha} \int_{E_{\nu_{\min }}}^{E_{\nu \max }} d E_{\nu} \int_{E_{T_{\min }}}^{E_{T_{\max }}} d E_{T} \int_{E_{A_{t h}}} d E_{A} \rho\left(E_{A}, E_{T}\right) X_{\alpha} \phi_{\alpha}\left(E_{\nu}\right) \\
& {\left[<P_{e e}\left(E_{\nu}\right)>_{\alpha} \frac{d \sigma_{\nu_{e}}}{d E_{T}}+<P_{e \mu}\left(E_{\nu}\right)>_{\alpha} \frac{d \sigma_{\nu_{\mu}}}{d E_{T}}\right] . }
\end{aligned}
$$


The second term in the bracket is absent if oscillation to sterile neutrinos is under consideration. $E_{T}$ and $E_{A}$ denote the true and apparent electron energies respectively. $E_{T_{\min }}$ and $E_{T_{\max }}$ are determined by kinematics. $\rho\left(E_{A}, E_{T}\right)$ is the energy resolution function for which we use the expression given in 25]. $E_{A_{t h}}$ is 7.5 (5.5) $\mathrm{MeV}$ for the calculation of the total rate at Kamiokande (SuperKamiokande). The differential cross-section for the production of an electron with true relativistic energy $E_{T}, \frac{d \sigma}{d E_{T}}$, is obtained from standard electroweak theory.

Now we discuss the results obtained from a $\chi^{2}$ minimization analysis of the data within the VEP picture. For these fits we have set $X_{\alpha}=1$ for all $\alpha$; i.e., we take the normalizations of the solar spectra at their SSM values. The best-fit parameters, $\chi_{\min }^{2} /$ (degree of freedom), and the goodness of fit values are presented in Table 2. Both possibilities of oscillation of the $\nu_{e}$ to an active or a sterile neutrino have been considered. To gauge the impact of the different experiments on the best-fit values of the VEP parameters, we have first fitted all the five data given in Table 1. We have then repeated the procedure excluding the Chlorine or the Kamiokande results. We also examine the effect of using the average of the result of the two Gallium measurements $(0.57 \pm 0.054)$ rather than the two individual ones. It is seen from Table 2 that in the different alternatives the best-fit values of the parameters are all rather close excepting for the case where the Chlorine result is excluded from the analysis. The fit improves significantly in the latter case; but even here the goodness of fit for active (sterile) neutrinos is still just $36 \%(58.5 \%)$. It is seen that maximal mixing is preferred and the VEP oscillation length is (see Eq. (5) $) \sim 3.4 \times 10^{11} \mathrm{~m}$, comparable with the earth-sun distance. This is reminiscent of the mass-mixing vacuum oscillation solution to the solar neutrino problem.

\begin{tabular}{|c|c|c|c|c|c|c|}
\hline $\begin{array}{c}\text { Neutrino } \\
\text { Type }\end{array}$ & Set & $\begin{array}{c}\text { Fitted } \\
\text { Experiments }\end{array}$ & $\sin ^{2} 2 \theta$ & $\begin{array}{c}\Delta F \\
\left(10^{-24}\right)\end{array}$ & $\chi_{\min }^{2} /$ d.o.f & $\begin{array}{c}\text { g.o.f } \\
(\%)\end{array}$ \\
\hline \multirow{5}{*}{ Active } & 1a & Cl, Gallex, SAGE, K, SK & 1.0 & 1.80 & $4.54 / 3$ & 20.84 \\
\cline { 2 - 7 } & 1b & Gallex, SAGE, K, SK & 0.85 & 4.57 & $2.04 / 2$ & 36.05 \\
\cline { 2 - 7 } & 1c & Cl, Gallex, SAGE, SK & 1.0 & 1.80 & $3.50 / 2$ & 17.41 \\
\cline { 2 - 7 } & $1 \mathrm{~d}$ & $\mathrm{Cl},(\mathrm{Ga})_{\mathrm{av}}, \mathrm{K}, \mathrm{SK}$ & 1.0 & 1.80 & $3.72 / 2$ & 15.59 \\
\cline { 2 - 7 } & $1 \mathrm{e}$ & $\mathrm{Cl},(\mathrm{Ga})_{\mathrm{av}}, \mathrm{SK}$ & 1.0 & 1.80 & $2.67 / 1$ & 10.20 \\
\hline \hline \multirow{5}{*}{ Sterile } & $2 \mathrm{a}$ & $\mathrm{Cl}, \mathrm{Gallex}, \mathrm{SAGE}, \mathrm{K}, \mathrm{SK}$ & 1.0 & 1.84 & $5.89 / 3$ & 11.73 \\
\cline { 2 - 7 } & 2b & Gallex, SAGE, K, SK & 0.84 & 4.53 & $1.07 / 2$ & 58.51 \\
\cline { 2 - 7 } & 2c & $\mathrm{Cl}, \mathrm{Gallex}, \mathrm{SAGE}, \mathrm{SK}$ & 1.0 & 1.84 & $4.58 / 2$ & 10.12 \\
\cline { 2 - 7 } & $2 \mathrm{~d}$ & $\mathrm{Cl},(\mathrm{Ga})_{\mathrm{av}}, \mathrm{K}, \mathrm{SK}$ & 1.0 & 1.84 & $5.08 / 2$ & 7.91 \\
\cline { 2 - 7 } & $2 \mathrm{e}$ & $\mathrm{Cl},(\mathrm{Ga})_{\mathrm{av}}, \mathrm{SK}$ & 1.0 & 1.84 & $3.77 / 1$ & 5.23 \\
\hline
\end{tabular}

Table 2: The best-fit values of the parameters, $\sin ^{2} 2 \theta, \Delta F, \chi_{m i n}^{2}$, and the g.o.f. for fits to the total rates of the different experiments.

As is seen from Table 2, the goodness of fits are rather poor for all the above cases. In order to trace the origin of this result, we present in Table 3 the total rates for the different experiments obtained using the best-fit values of the VEP parameters presented 
in Table 2. These should be compared with the experimental data in Table 1. Several points are noteworthy. The rates for the different experiments are not sensitive to the small changes in the input VEP parameters; the numbers for the Gallium experiments and Kamiokande are always within $1 \sigma$ of the experimental value but for Chlorine the deviation is $3 \sigma$. The fit to SuperKamiokande is always bad $(8 \sigma)$ irrespective of which experiments are excluded from the analysis excepting for the singular case where the Chlorine rate is left out when a $2 \sigma$ fit is obtained. This is a reflection of the very precise nature of the present SK data and the inability of the VEP mechanism to simultaneously reproduce the varying degrees of suppression seen in experiments with different energy thresholds. In particular, the Chlorine result is seen to be especially problematic in this respect. It can be surmised from the Chlorine-excluded analyses - Table 3 (sets 1b, 2b) - that a simultaneous good fit to all data would be obtained if the suppression in the Chlorine experiment had been less than that in the Gallium experiments (lower threshold) as well as in the water Cerenkov ones (higher threshold), indicating that for these fit-values of the VEP parameters the ${ }^{7} \mathrm{Be}$ neutrinos are less suppressed than the other solar neutrinos.

\begin{tabular}{|c|c|c|c|c|c|c|}
\hline $\begin{array}{c}\text { Neutrino } \\
\text { Type }\end{array}$ & Set & $\begin{array}{c}\text { Fitted } \\
\text { Experiments }\end{array}$ & Cl & Ga & K & SK \\
\hline \multirow{5}{*}{ Active } & $1 \mathrm{a}$ & $\mathrm{Cl}$, Gallex, SAGE, K, SK & 0.436 & 0.572 & 0.600 & 0.600 \\
\cline { 2 - 7 } & $1 \mathrm{~b}$ & Gallex, SAGE, K, SK & $0.655^{*}$ & 0.549 & 0.508 & 0.497 \\
\cline { 2 - 7 } & $1 \mathrm{c}$ & $\mathrm{Cl}, \mathrm{Gallex}, \mathrm{SAGE}, \mathrm{SK}$ & 0.436 & 0.572 & $0.600^{*}$ & 0.600 \\
\cline { 2 - 7 } & $1 \mathrm{~d}$ & $\mathrm{Cl},(\mathrm{Ga})_{\mathrm{av}}, \mathrm{K}, \mathrm{SK}$ & 0.436 & 0.574 & 0.600 & 0.600 \\
\cline { 2 - 7 } & $1 \mathrm{e}$ & $\mathrm{Cl},(\mathrm{Ga})_{\mathrm{av}}, \mathrm{SK}$ & 0.436 & 0.574 & $0.600^{*}$ & 0.600 \\
\hline \hline \multirow{5}{*}{ Sterile } & $2 \mathrm{a}$ & $\mathrm{Cl}, \mathrm{Gallex}, \mathrm{SAGE}, \mathrm{K}, \mathrm{SK}$ & 0.435 & 0.565 & 0.525 & 0.525 \\
\cline { 2 - 7 } & $2 \mathrm{~b}$ & $\mathrm{Gallex}, \mathrm{SAGE}, \mathrm{K}, \mathrm{SK}$ & $0.657^{*}$ & 0.555 & 0.512 & 0.468 \\
\cline { 2 - 7 } & 2c & $\mathrm{Cl}, \mathrm{Gallex}, \mathrm{SAGE}, \mathrm{SK}$ & 0.435 & 0.565 & $0.525^{*}$ & 0.525 \\
\cline { 2 - 7 } & $2 \mathrm{~d}$ & $\mathrm{Cl},(\mathrm{Ga})_{\mathrm{av}}, \mathrm{K}, \mathrm{SK}$ & 0.435 & 0.565 & 0.525 & 0.525 \\
\cline { 2 - 7 } & $2 \mathrm{e}$ & $\mathrm{Cl},(\mathrm{Ga})_{\mathrm{av}}, \mathrm{SK}$ & 0.435 & 0.565 & $0.525^{*}$ & 0.525 \\
\hline
\end{tabular}

Table 3: The total rate predictions for the different experiments obtained by using the best-fit values of the VEP parameters presented in Table 2. Predictions for experiments not included in the fit are marked with an asterisk.

We show in Fig. 1, the allowed region for the parameters $\sin ^{2} 2 \theta$ and $\Delta F$ at $90 \%$ C.L. for the (1a) active and (1b) sterile cases obtained by fitting the total rates of all five experiments, i.e., Chlorine, Gallex, SAGE, Kamiokande, and SuperKamiokande. The confidence level has been fixed with respect to the global minimum. The best fit points have been indicated. It is seen from Fig. 1 that the nature of the allowed regions for the active and sterile cases are roughly similar, an observation made by Gago et al. [19]. The sterile alternative is more restrictive. 


\section{SuperKamiokande Recoil electron energy spectrum and VEP}

We now turn to a fit to the recoil electron energy spectrum as seen at SuperKamiokande. We use the 1117-day data for this analysis. The SK results have been presented in the form of number of events in 17 electron recoil energy bins of width $0.5 \mathrm{MeV}$ in the range $5.5 \mathrm{MeV}$ to $14 \mathrm{MeV}$ and an 18th bin which covers the events in the range 14 to $20 \mathrm{MeV}$ [1, 26].

With the SuperKamiokande threshold in the $5 \mathrm{MeV}$ range, only the ${ }^{8} \mathrm{~B}$ and hep solar neutrinos contribute to the signal. In addition to the SSM, for this analysis we have examined models in which the normalizations of both the ${ }^{8} \mathrm{~B}$ and hep neutrino flux $\left(X_{B}\right.$ and $X_{h}$, respectively - normalized to unity for the SSM) are allowed to vary arbitrarily. In this case, $\chi^{2}$ is defined as

$$
\chi^{2}=\sum_{i, j=1,18}\left(R_{i}^{t h}-R_{i}^{e x p}\right)\left(\sigma_{i j}^{-2}\right)_{s p}\left(R_{j}^{t h}-R_{j}^{e x p}\right) .
$$

$R_{i}^{\xi}=S_{i}^{\xi} / S_{i}^{B P 98}$ with $\xi$ being th or exp as before and $S_{i}$ standing for the number of events in the $i$-th energy bin. The theoretical prediction is given by eq. (11) but the integration over the apparent (i.e., measured) energy will now be over each bin. The error matrix $\sigma_{i j}$ used by us is $[7$

$$
\left(\sigma_{i j}^{2}\right)_{s p}=\delta_{i j}\left(\sigma_{i, \text { stat }}^{2}+\sigma_{i, \text { uncorr }}^{2}\right)+\sigma_{i, \exp } \sigma_{j, \exp }+\sigma_{i, \text { cal }} \sigma_{j, \text { cal }},
$$

where we have included the statistical error, the uncorrelated systematic errors and the energy-bin-correlated experimental errors [27] as well as those from the calculation of the shape of the expected spectrum [28]. Since we allow the normalizations of the ${ }^{8} \mathrm{~B}$ and hep fluxes to vary, we do not include their astrophysical uncertainties separately. The results are presented in Table 4.

\begin{tabular}{|c|c|c|c|c|c|c|c|}
\hline $\begin{array}{c}\text { Neutrino } \\
\text { Type }\end{array}$ & Set & $\sin ^{2} 2 \theta$ & $\begin{array}{c}\Delta F \\
\left(10^{-24}\right)\end{array}$ & $X_{B}$ & $X_{h}$ & $\chi_{\min }^{2} /$ d.o.f. & $\begin{array}{c}\text { g.o.f } \\
(\%)\end{array}$ \\
\hline \multirow{5}{*}{ Active } & $3 \mathrm{a}$ & 1.0 & $1.97 \times 10^{2}$ & 0.79 & 15.07 & $8.83 / 14$ & 84.2 \\
\cline { 2 - 8 } & $3 \mathrm{~b}$ & 0.38 & 0.22 & 0.65 & $\begin{array}{c}1.0 \\
\text { (fixed) }\end{array}$ & $10.04 / 15$ & 81.7 \\
\cline { 2 - 8 } & $3 \mathrm{c}$ & 0.69 & 0.23 & $\begin{array}{c}1.0 \\
\text { (fixed) }\end{array}$ & -2.20 & $11.59 / 15$ & 70.97 \\
\cline { 2 - 8 } & $3 \mathrm{~d}$ & 0.68 & 0.23 & $\begin{array}{c}1.0 \\
\text { (fixed) }\end{array}$ & $\begin{array}{c}1.0 \\
\text { (fixed) }\end{array}$ & $11.66 / 16$ & 76.7 \\
\hline \hline \multirow{5}{*}{ Sterile } & $4 \mathrm{a}$ & 1.0 & 39.33 & 1.03 & 11.52 & $8.78 / 14$ & 84.48 \\
\cline { 2 - 8 } & $4 \mathrm{~b}$ & 0.34 & 0.22 & 0.67 & 1.0 & $10.01 / 15$ & 81.9 \\
\cline { 2 - 8 } & $4 \mathrm{c}$ & 0.98 & 39.33 & $\begin{array}{c}1.0 \\
\text { (fixed) }\end{array}$ & 11.94 & $8.81 / 15$ & 88.71 \\
\cline { 2 - 8 } & $4 \mathrm{~d}$ & 0.58 & 0.23 & $\begin{array}{c}1.0 \\
\text { (fixed) }\end{array}$ & $\begin{array}{c}1.0 \\
\text { (fixed) }\end{array}$ & $11.34 / 16$ & 78.8 \\
\hline
\end{tabular}


Table 4: The best-fit values of the parameters, $\sin ^{2} 2 \theta, \Delta F, X_{B}, X_{h}, \chi_{\min }^{2}$, and the g.o.f. for fits to the scattered electron spectrum at SK.

The large values of $X_{h}$ obtained from the fits with the best g.o.f. (3a and $4 \mathrm{~b}$ ) indicate that an increased number of high energy hep neutrinos yields a better fit to the highest energy bin of the spectrum observed at SK. It may be noteworthy that a rise in the observed electron energy spectrum at the high energy end seen in the earlier 825-day data has become less prominent in the latest 1117-day sample. A further softening will bring $X_{h}$ closer to the SSM prediction?.

\begin{tabular}{|c|c|c|c|c|c|c|c|c|c|}
\hline $\begin{array}{c}\text { Neutrino } \\
\text { Type }\end{array}$ & Set & $\sin ^{2} 2 \theta$ & $\begin{array}{c}\Delta F \\
\left(10^{-24}\right)\end{array}$ & $X_{B}$ & $X_{h}$ & $\mathrm{Cl}$ & $\mathrm{Ga}$ & $\mathrm{K}$ & $\overline{S K}$ \\
\hline \multirow{4}{*}{ Active } & $3 a$ & 1.0 & $\begin{array}{c}1.97 \\
\times 10^{2}\end{array}$ & 0.79 & 15.07 & 0.412 & 0.463 & 0.471 & 0.466 \\
\hline & $3 \mathrm{~b}$ & 0.38 & 0.22 & 0.65 & $\begin{array}{c}1.0 \\
\text { (fixed) }\end{array}$ & 0.585 & 0.947 & 0.481 & 0.488 \\
\hline & $3 \mathrm{c}$ & 0.69 & 0.23 & $\begin{array}{c}1.0 \\
\text { (fixed) }\end{array}$ & -2.20 & 0.560 & 0.944 & 0.497 & 0.503 \\
\hline & $3 \mathrm{~d}$ & 0.68 & 0.23 & $\begin{array}{c}1.0 \\
\text { (fixed) }\end{array}$ & $\begin{array}{c}1.0 \\
\text { (fixed) }\end{array}$ & 0.562 & 0.944 & 0.507 & 0.513 \\
\hline \multirow{4}{*}{ Sterile } & $4 a$ & 1.0 & 39.33 & 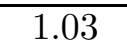 & 11.52 & 0.562 & 0.538 & 0.479 & 0.477 \\
\hline & $4 \mathrm{~b}$ & 0.34 & 0.22 & 0.67 & $\begin{array}{c}1.0 \\
\text { (fixed) }\end{array}$ & $\overline{0.612}$ & 0.951 & 0.485 & 0.492 \\
\hline & $4 \mathrm{c}$ & 0.98 & 39.33 & $\begin{array}{c}1.0 \\
\text { (fixed) }\end{array}$ & 11.94 & 0.563 & 0.548 & 0.477 & 0.476 \\
\hline & $4 \mathrm{~d}$ & 0.58 & 0.23 & $\begin{array}{c}1.0 \\
\text { (fixed) }\end{array}$ & $\begin{array}{c}1.0 \\
\text { (fixed) }\end{array}$ & 0.630 & 0.953 & 0.507 & 0.509 \\
\hline
\end{tabular}

Table 5: The calculated values of the rates for the different experiments using the best-fit values of the parameters, $\sin ^{2} 2 \theta, \Delta F, X_{B}$, and $X_{h}$, from fits to the scattered electron spectrum at SK.

It may be of interest to check how the best-fit values of the VEP parameters fare when confronted with the total rates data. For this purpose, we use the four sets of best-fit values of parameters from Table 4 and use them to compute the total rates from the different experiments. These results are shown in Table 5. The latter may be compared to the values obtained using fits to the total rates themselves (see Table 3). Using Table 1, it is seen that the predicted rate for SK is in rather close agreement with the measured value, as is to be expected, but for the Chlorine and Gallium experiments the deviations are very large. Notice, in particular, that in several cases only a very tiny suppression is obtained for the Gallium experiments, though for the fits to the spectrum with the best g.o.f. - fits 3a and 4c (see Table 4) - this is not the case. This underscores a basic incompatibility, within the VEP picture, of the results from the different experiments.

\footnotetext{
${ }^{5}$ See the remarks on fits to the SK separate day and night spectra measurements in section VII
} 
In Fig. 2 we show the $90 \%$ C.L. allowed region in the $\sin ^{2} 2 \theta-\Delta F$ plane obtained by fitting the scattered electron spectrum for the $X_{B}=X_{h}=1$ case (area enclosed by the solid lines). The best-fit point is marked with a dark dot. Also shown is the $90 \%$ C.L. disallowed region when $X_{B}$ is permitted to float arbitrarily but keeping $X_{h}$ fixed at unity (area enclosed by the broken lines). The best-fit point in this case has been indicated by a $\times$ sign. Both (a) active and (b) sterile neutrino alternatives have been displayed.

\section{Combined analysis of rates and spectrum in the VEP sce- nario}

In the previous sections we have examined the solar neutrino total rates and the SK scattered electron energy spectrum within the VEP oscillation framework. For the latter, we found a good fit using SSM input parameters and even better ones when the absolute normalizations of the ${ }^{8} \mathrm{~B}$ and/or hep fluxes are allowed to vary (see Table 4). The rates fit, on the other hand, was not satisfactory and improved somewhat if the results from the Chlorine experiment was left out from the $\chi^{2}$ analysis. In this section we make a combined fit to the rates and spectrum data.

For the combined analysis of rate and spectrum, we take the rate measurements from the Chlorine experiment, the average of the Gallex and SAGE results, and the SK 1117-day rate data. To obtain the $\chi^{2}$ corresponding to any value of the input parameters, separate values of $\chi^{2}$ calculated for the total rates and the spectrum data are added and then the total is minimized. The best-fit values for VEP oscillations to active and sterile neutrinos are given in Table 6 . The number of degrees of freedom for this case is $(18+3-4)=17$. Notice that good fits (cases $5 \mathrm{a}$ and $6 \mathrm{a}$ ) can be obtained only when both $X_{B}$ and $X_{h}$ are allowed to assume values different from their SSM requirements.

\begin{tabular}{|c|c|c|c|c|c|c|c|}
\hline $\begin{array}{c}\text { Neutrino } \\
\text { Type }\end{array}$ & Set & $\sin ^{2} 2 \theta$ & $\begin{array}{c}\Delta F \\
\left(10^{-24}\right)\end{array}$ & $X_{B}$ & $X_{h}$ & $\chi_{\min }^{2} /$ d.o.f. & $\begin{array}{c}\text { g.o.f } \\
(\%)\end{array}$ \\
\hline \multirow{5}{*}{ Active } & $5 \mathrm{a}$ & 1.0 & 1.72 & 0.79 & 23.69 & $11.51 / 17$ & 82.87 \\
\cline { 2 - 8 } & $5 \mathrm{~b}$ & 1.0 & 1.68 & 0.79 & $\begin{array}{c}1.0 \\
\text { (fixed) }\end{array}$ & $12.96 / 18$ & 79.38 \\
\cline { 2 - 8 } & $5 \mathrm{c}$ & 1.0 & 19.26 & $\begin{array}{c}1.0 \\
\text { (fixed) }\end{array}$ & 33.57 & $50.79 / 15$ & $8.9 \times 10^{-4}$ \\
\cline { 2 - 8 } & $5 \mathrm{~d}$ & 1.0 & 18.75 & $\begin{array}{c}1.0 \\
\text { (fixed) }\end{array}$ & $\begin{array}{c}1.0 \\
\text { (fixed) }\end{array}$ & $70.44 / 16$ & $8.37 \times 10^{-7}$ \\
\hline \hline \multirow{3}{*}{ Sterile } & $6 \mathrm{a}$ & 1.0 & 1.77 & 0.91 & 34.55 & $14.04 / 17$ & 66.45 \\
\cline { 2 - 8 } & $6 \mathrm{~b}$ & 1.0 & 1.86 & 0.91 & $\begin{array}{c}1.0 \\
\text { (fixed) }\end{array}$ & $16.28 / 18$ & 57.28 \\
\cline { 2 - 8 } & $6 \mathrm{c}$ & 1.0 & 1.86 & $\begin{array}{c}1.0 \\
\text { (fixed) }\end{array}$ & 36.87 & $26.90 / 15$ & 2.95 \\
\cline { 2 - 8 } & $6 \mathrm{~d}$ & 1.0 & 1.89 & $\begin{array}{c}1.0 \\
\text { (fixed) }\end{array}$ & $\begin{array}{c}1.0 \\
\text { (fixed) }\end{array}$ & $29.84 / 16$ & 1.88 \\
\hline
\end{tabular}


Table 6: The best-fit values of the parameters, $\sin ^{2} 2 \theta, \Delta F, X_{B}, X_{h}, \chi_{\min }^{2}$, and the g.o.f. for fits to the total rates as well as the scattered electron spectrum at SK.

In exhibiting the $90 \%$ C.L. allowed regions in parameter space from this combined fit, we present only the results for the $X_{h}=1, X_{B}$ arbitrary case. As already noted, if the SSM normalization for the ${ }^{8} \mathrm{~B}$ flux is used $\left(X_{B}=1\right)$ then the goodness-of-fits are poor. These cases are not pursued further. In Fig. 3 we present the $90 \%$ C.L. allowed regions in the $\sin ^{2} 2 \theta-\Delta F$ plane from a combined analysis of total rates and spectrum. The best-fit points are marked by a $\times$ sign. Both $(\mathrm{a})$ active and (b) sterile neutrino alternatives have been considered. It is noteworthy that the allowed regions have a considerable overlap with that in Fig. 1 obtained from a fit to the total rates alone.

\section{Predictions for SNO}

The analyses described in the previous sections yield sets of best-fit values for the parameters $\sin ^{2} 2 \theta, \Delta F, X_{B}$ and $X_{h}$. In this section we examine what these best-fit values imply for the Sudbury Neutrino Observatory (SNO) experiment [29].

At SNO the neutrinos are detected by three processes, namely, (a) charge current (CC) break up of the deuteron, (b) electron scattering by the neutrino, and (c) neutral current $(\mathrm{NC})$ break up of the deuteron.

$$
\begin{array}{rlr}
\nu_{e}+d & \rightarrow p+p+e^{-} & (\mathrm{CC} \text { reaction }) \\
\nu+e^{-} & \rightarrow \nu+e^{-} & (\text {scattering }) \\
\nu+d & \rightarrow \nu+p+n & (\mathrm{NC} \text { reaction }) .
\end{array}
$$

For the scattering (15) and NC (16) reactions, $\nu$ stands for any active neutrino; it has to be borne in mind that for the $\mu$ and $\tau$ flavours only the $Z$ exchange contribution is present for the former reaction while for the $\nu_{e}$ there is an additional (dominant) piece from $W$ exchange. For the $\mathrm{CC}$ reaction and for scattering, the electrons are detected by the emitted Cerenkov radiation and hence their energy spectrum is directly measured as at SK. For the $\mathrm{NC}$ reaction, on the other hand, only calorimetric measurment is possible. At present, data is being taken for the first two processes.

For the scattered electrons, the formalism for theoretical prediction is similar to that for SK (see eq. (11)) except for the fact that here in SNO, the detector fluid is $1 \mathrm{~K}$ ton of $\mathrm{D}_{2} \mathrm{O}$ instead of $32 \mathrm{~K}$ ton of water in SK.

For the $\mathrm{CC}$ interactions, the theoretical predictions for the electron energy spectrum at $\mathrm{SNO}$ is given by

$$
T_{S N O}^{t h}=\sum_{\alpha} \int_{E_{\nu_{\min }}}^{E_{\nu_{\max }}} d E_{\nu} \int d E_{T} \int_{E_{A_{t h}}} d E_{A} \rho\left(E_{A}, E_{T}\right) X_{\alpha} \phi_{\alpha}\left(E_{\nu}\right)<P_{e e}\left(E_{\nu}\right)>_{\alpha} \sigma^{C C} .
$$

The charge current scattering cross-section at SNO, $\sigma^{C C}$, is found from Ref. 30]. In our calculations, we use the resolution function $\rho\left(E_{A}, E_{T}\right)$ for SNO as given in 225]. We have set $E_{A_{t h}}$ to $5.0 \mathrm{MeV}$. 
In Fig. 4 we show the signal expected at SNO due to (a) $\nu-e$ scattering and (b) the $\mathrm{CC}$ reaction if the VEP oscillation is operative. We choose two typical cases for the VEP parameters: (i) the best-fit values obtained from the fit to the spectrum using the SSM inputs $\left(X_{B}=X_{h}=1\right)$, and (ii) the best-fit parameters from the combined rate and spectrum fit when both $X_{B}$ and $X_{h}$ are allowed to vary. The SSM expectation in the absence of VEP transitions is also shown.

Figs 5(a) and 5(b) are similar to Figs. 4(a) and 4(b) excepting that we present the expectations at SNO normalized by the SSM predictions. This form may be of more convenience for comparison with the experimental results.

SNO will subsequently also detect neutrinos via the NC reaction (16). Since all active neutrinos register in the $\mathrm{NC}$ reaction with the same strength, this reaction provides a means to distinguish oscillations to active neutrinos from those to sterile ones. The ratio of the $\mathrm{NC}$ and CC rates, $R_{N C}$ and $R_{C C}$, is somewhat less sensitive to theoretical uncertainties than the rates themselves. Therefore, for the purpose of illustration, we present in Table 7 the values for the ratios

$$
\begin{aligned}
R_{S N O} & =\frac{R_{N C}}{R_{C C}}, \\
S_{S N O} & =R_{S N O} / R_{S N O}^{B P 98}=\frac{R_{N C} / R_{N C}^{B P 98}}{R_{C C} / R_{C C}^{B P 98}} .
\end{aligned}
$$

For the NC cross-sections we use Ref. [30]. Results are presented for the best-fit values of the parameters obtained from the total rates, the SK scattered electron spectrum, and the combined rates and spectrum data presented in Tables 2, 4, and 6 .

It is seen from Table 7 that the variables $R_{S N O}$ and $S_{S N O}$ are particularly effective in distinguishing VEP oscillations to active neutrinos from those to a sterile neutrino. For active neutrinos $R_{S N O}\left(S_{S N O}\right)$ varies between $0.44-0.74(1.15-1.92)$ while the corresponding range for sterile neutrinos is $0.29-0.30(0.77-0.79)$. 


\begin{tabular}{|c|c|c|c|c|c|c|c|}
\hline $\begin{array}{c}\text { Neutrino } \\
\text { Type }\end{array}$ & Set & $\sin ^{2} 2 \theta$ & $\begin{array}{c}\Delta F \\
\left(10^{-24}\right)\end{array}$ & $X_{B}$ & $X_{h}$ & $R_{S N O}$ & $S_{S N O}$ \\
\hline \multirow{7}{*}{ Active } & 1a,c,d,e & 1.0 & 1.80 & 1.0 & 1.00 & 0.610 & 1.57 \\
\hline & $1 \mathrm{~b}$ & 0.85 & 4.57 & 1.0 & 1.00 & 0.740 & 1.92 \\
\hline & $3 a$ & 1.0 & $1.97 \times 10^{2}$ & 0.79 & 15.07 & 0.581 & 1.50 \\
\hline & $3 \mathrm{~b}$ & 0.38 & 0.22 & 0.65 & $\begin{array}{c}1.0 \\
\text { (fixed) }\end{array}$ & 0.443 & 1.15 \\
\hline & $3 c$ & 0.69 & 0.23 & $\begin{array}{c}1.0 \\
\text { (fixed) }\end{array}$ & -2.20 & 0.724 & 1.87 \\
\hline & $3 \mathrm{~d}$ & 0.68 & 0.23 & $\begin{array}{c}1.0 \\
\text { (fixed) }\end{array}$ & $\begin{array}{c}1.0 \\
\text { (fixed) }\end{array}$ & 0.712 & 1.89 \\
\hline & $5 a$ & 1.0 & 1.72 & 0.79 & 23.69 & 0.583 & 1.51 \\
\hline \multirow{7}{*}{ Sterile } & 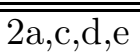 & $\overline{1.0}$ & 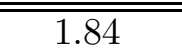 & $\bar{~} 1.0$ & $\overline{1.00}$ & 0.305 & $\overline{0.789}$ \\
\hline & $2 \mathrm{~b}$ & 0.84 & 4.53 & 1.0 & 1.00 & 0.303 & 0.784 \\
\hline & $4 a$ & 1.0 & 39.33 & 1.03 & 11.52 & 0.300 & 0.776 \\
\hline & $4 \mathrm{~b}$ & 0.34 & 0.22 & 0.67 & $\begin{array}{c}1.0 \\
\text { (fixed) }\end{array}$ & 0.299 & 0.774 \\
\hline & $4 c$ & 0.98 & 39.33 & $\begin{array}{c}1.0 \\
\text { (fixed) }\end{array}$ & 11.94 & 0.299 & 0.775 \\
\hline & $4 d$ & 0.58 & 0.23 & $\begin{array}{c}1.0 \\
\text { (fixed) }\end{array}$ & $\begin{array}{c}1.0 \\
\text { (fixed) }\end{array}$ & 0.302 & 0.781 \\
\hline & $6 a$ & 1.0 & 1.77 & 0.91 & 34.55 & 0.302 & 0.781 \\
\hline
\end{tabular}

Table 7: The ratio of the NC rate to the $\mathrm{CC}$ rate at $\mathrm{SNO}, R_{S N O}$, and the same ratio normalized to the BP98 SSM, $S_{S N O}$ for the best-fit values of the parameters, $\sin ^{2} 2 \theta, \Delta F, X_{B}, X_{h}$ obtained from fits to the total rates and the SK scattered electron spectrum. The sets 1 and 2 are from Table 2, 3 and 4 from Table 4 , and 5 and 6 from Table 6.

\section{Discussions and Conclusions}

In this work we have made a detailed examination of the viability of the VEP oscillation mechanism in the light of the solar neutrino data. The parameters in the VEP formalism are $\Delta F$, a measure of the violation of the weak equivalence principle, and $\theta$, the mixing angle relating the flavor basis of neutrinos to the gravitational basis. The data we have included in the analysis come from the radiochemical Chlorine experiment, the similar Galliumbased Gallex and SAGE collaborations, and the Cerenkov technique reliant Kamiokande and SuperKamiokande (1117-day data) facilities. We have found that good fits $\left(\chi^{2} /\right.$ d.o.f. $=11.66 / 16$ for active neutrinos) can be obtained to the scattered electron energy spectrum seen at SK within the VEP mechanism using SSM inputs. The fits are even better in models in which the absolute normalizations of the ${ }^{8} \mathrm{~B}$ and/or hep fluxes are allowed to vary. The preferred oscillation wavelength is comparable to the earth-sun distance and is reminiscent of the mass-mixing vacuum oscillation solution. In contrast to the fit to the spectral data, the fit to the observed rates is poor but improves somewhat $\left(\chi^{2} /\right.$ d.o.f. $=2.04 / 2$ for active 
neutrinos) if the Chlorine experiment is left out of the fit. A combined fit to the rates and spectrum has also been performed and it is found that within the SSM the goodness is not satisfactory. However, in a model in which the absolute normalizations of the ${ }^{8} \mathrm{~B}$ and hep fluxes are allowed to vary, the fit is much improved $\left(\chi^{2} /\right.$ d.o.f. $=11.51 / 17$ for active neutrinos with $X_{B}=0.79$ and $\left.X_{h}=23.69\right)$. The best-fit values of $\Delta F\left(=1.7 \times 10^{-24}\right)$ and $\sin ^{2} 2 \theta(=1)$ are closer to those obtained from the fits to the rates alone. We find that the 90\% C.L. allowed regions are broadly similar whether VEP transitions occur to active or sterile neutrinos.

It is of interest to compare the results that we have obtained with those of other recent analyses of the solar neutrino problem in the VEP picture [19, 20]. For the fit to the total rates, our results agree almost exactly with those of [20] and are within $5 \%$ of those of [19]. The earlier analyses used the 825-day SK results and we must therefore conclude that the newer data do not make a significant impact on this fit. In contrast, for the fits to the SK recoil electron energy spectrum, our best-fit values of $\Delta F$ and $\sin ^{2} 2 \theta$ are both somewhat smaller than those of [19, 20]. We have checked that if we use the 825-day SK data there is better agreement. We attribute the difference to the reduction of the higher energy events in the newer data. For the combined fit to the rate and spectrum, we are in good agreement with [19]; the goodness-of-fit has improved with the newer data.

During the passage of the neutrinos from their point of production to the solar surface, interactions with the ambient matter, responsible for the MSW effect, become important. Apart from a neutral current contribution which affects the masses of all active neutrino species identically (and is therefore irrelevant for this discussion), there is a contribution to the electron neutrino mass $m_{M S W} \simeq \sqrt{2} G_{F} n_{e}(r), G_{F}$ being the Fermi coupling and $n_{e}(r)$ the number density of electrons at a distance $r$ from the center of the sun, due to charge current interactions. In the presence of VEP and this MSW contribution, the effective neutrino mass matrix in flavor space takes the form

$$
M=\frac{1}{2}\left|\begin{array}{cc}
E_{\nu} \Delta F \cos 2 \theta-2 \sqrt{2} G_{F} n_{e}(r) & E_{\nu} \Delta F \sin 2 \theta \\
E_{\nu} \Delta F \sin 2 \theta E_{\nu} & -E_{\nu} \Delta F \cos 2 \theta
\end{array}\right|,
$$

where we have dropped an irrelevant part proportional to the identity matrix. The MSW contribution in (19) inside the sun turns out to be several orders of magnitude larger than the terms due to VEP that we have discussed in this work. Recall that we have assumed the neutrinos to be massless. For the maximal mixing case (i.e., $\sin 2 \theta=1$ ), which we have found for the best-fit solutions, there is no resonance effect and, in fact, till such time that the neutrino emerges from the sun, the MSW contribution controls the masses in (19). Inside the sun, the $\nu_{e}$ is, therefore, a mass eigenstate to a very good approximation. The effect of VEP oscillations begins to manifest itself only from then onwards. For oscillation to a sterile neutrino, the neutral current contribution to the $\nu_{e}$ mass also becomes relevant. It is of the same order as the charged current piece and the resultant effect in this case is much the same as that for the active neutrino alternative. There is a different region in the $\sin ^{2} 2 \theta, \Delta F$ parameter space (larger $\Delta F$, non-maximal mixing) where other solutions have been found with the MSW effect playing an important role [16, 18].

\footnotetext{
${ }^{6}$ It has, however, been recently demonstrated that these solutions are inconsistent with the results from
} 
SuperKamiokande has now obtained the recoil electron spectra separately for day and night runs using their 1117-day data [31]. The oscillation wavelengths corresponding to the best-fit VEP parameters that we have found are comparable to the earth-sun distance (1.49 $\left.\times 10^{11} \mathrm{~m}\right)$. The extra distance travelled by neutrinos through the earth during the night runs is insignificant compared to this. This distance can be readily estimated. Using the latitude of SuperKamiokande $\left(36.4^{\circ}\right)$ and the obliquity of the ecliptic $\left(23.5^{\circ}\right)$, we estimate the maximum extra distance travelled during the night run to be $6.40 \times 10^{6} \mathrm{~m}$. To confirm the expectation that fits to the separate day and night spectra give results which are not much different, we performed a $\chi^{2}$-minimization on this data for oscillation to an active neutrino. When $X_{B}$ and $X_{h}$ were held fixed at their SSM values of unity, the best fit corresponds to $\sin ^{2} 2 \theta=0.71$ and $\Delta F=0.21 \times 10^{-24}$. For comparison, the corresponding values from Table 4 , set $3 \mathrm{~d}$ are $\sin ^{2} 2 \theta=0.68$ and $\Delta F=0.23 \times 10^{-24}$. If $X_{B}$ and $X_{h}$ are allowed to vary then we get $\sin ^{2} 2 \theta=1.0(1.0), \Delta F=1.97 \times 10^{-22}\left(1.97 \times 10^{-22}\right)$, $X_{B}=0.78(0.79)$, and $X_{h}=0.74 \times 10^{-2}(15.07)$. In the parantheses we have given the values presented earlier in Table 4 , set $3 \mathrm{a}$. Notice that there is no significant change barring the very small value of $X_{h}$. The $X_{h}$ dependence of $\chi^{2}$ is slow and this indicates that the day-night scattered electron spectra is consistent with no hep neutrinos.

The VEP oscillation wavelengths favored by the data are comparable to the distance of the sun to the earth. Therefore, seasonal effects are to be expected if this mechanism is responsible for the solar neutrino deficit. However, the data from SK is still not of very high statistics and we have left this analysis to a future work.

\section{Acknowledgements}

The authors are grateful to the Calcutta University Computer Centre for the use of their Origin 2000 computer. D.M. and A.R. were partially supported by the Eastern Centre for Research in Astrophysics, India. A.R. also acknowledges a research grant from CSIR, India. A.S. was supported by a research grant from BRNS, India and now enjoys a fellowship from UGC, India.

\section{References}

[1] Y. Suzuki, Talk given at the XIX International Conference on Neutrino Physics \& Astrophysics, http://nu2000.sno.laurentian.ca/Y.Suzuki.

[2] Y. Fukuda et al., (The Super-Kamiokande collaboration), Phys Rev. Lett. 81, 1158 (1998), 82, 2430 (1999).

[3] B.T. Cleveland et al., Astrophys. J. 496, 505 (1998); Nucl. Phys. B (Proc. Suppl.) 38, 47 (1995); Y. Fukuda et al., (The Kamiokande collaboration), Phys. Rev. Lett. 77, 1683

the non-observation of oscillations in accelerator neutrino experiments 32 . 
(1996); W. Hampel et al., (The Gallex collaboration), Phys. Lett. B447, 127 (1999); J.N. Abdurashitov et al., (The SAGE collaboration), Phys. Rev. C60, 055801 (1999).

[4] Y. Fukuda et al., (The Super-Kamiokande collaboration), Phys. Rev. Lett. 81, 1562 (1998).

[5] L. Wolfenstein, Phys. Rev. D34, 969 (1986); S.P. Mikheyev and A.Yu. Smirnov, Sov. J. Nucl. Phys. 42(6), 913 (1985); Nuovo Cimento 9c, 17 (1986).

[6] J.N. Bahcall, P.I. Krastev, and A.Yu. Smirnov, Phys. Rev. D58, 096016 (1998).

[7] M.C. Gonzalez-Garcia, P.C. de Holanda, C. Peña-Garay, and J.W.F. Valle, hepph/9906469, Nucl. Phys. B573, 3 (2000).

[8] J.N. Bahcall, P.L. Krastev, and A.Yu. Smirnov, Phys. Lett. B477, 401 (2000).

[9] G.L. Fogli et al., hep-ph/9912231, Phys. Rev. D62, 013002 (2000).

[10] C. Giunti, M.C. Gonzalez-Garcia, and C. Peña-Garay, hep-ph/0001101, Phys. Rev. D62, 013005 (2000).

[11] S. Goswami, D. Majumdar, and A. Raychaudhuri, hep-ph/0003163 and work in progress.

[12] M. Gasperini, Phys. Rev. D38, 2635 (1988); 39, 3606 (1989); A. Halprin and C.N. Leung, Phys. Rev. Lett. 67, 1833 (1991).

[13] K. Iida, H. Minakata, and O. Yasuda, Mod. Phys. Lett. A8, 1037 (1993); J. Pantaleone, A. Halprin, and C.N. Leung, Phys. Rev. D47, R4199 (1993); M.N. Butler, S. Nazawa, R. Malaney, and A.I. Boothroyd, ibid. 47, 2615 (1993); H. Minakata and H. Nunokawa, ibid. 51, 6625 (1995); R.B. Mann and U.Sarkar, Phys. Rev. Lett. 76, 865 (1996); J.R. Mureika and R.B. Mann, Phys. Rev. D54, 2761 (1996).

[14] S. Coleman, S.L. Glashow, Phys. Lett. B405, 249 (1997); S.L. Glashow, A. Halprin, et al., Phys. Rev. D56, 2433 (1997).

[15] S. Coleman and S.L. Glashow, Phys. Rev. D59, 116008 (1999). For earlier work, see Colladay and V.A. Kostelecky, ibid. D55, 6760 (1997), D58, 116002 (1998).

[16] J.N. Bahcall, P.I. Krastev, C.N. Leung, Phys. Rev. D52, 1770 (1995); A. Halprin, C.N. Leung, and J. Pantaleone, ibid. 53, 5365 (1996).

[17] H. Minakata, H. Nunokawa, Phys. Rev. D51, 6625 (1995).

[18] S.W. Mansour and T.K. Kuo, Phys. Rev. D60, 097301 (1999).

[19] A.M. Gago, H. Nunokawa, R. Zukanovich Funchal, e-print no. hep-ph/9909250; Phys. Rev. Lett. 84, 4035 (2000). 
[20] H. Casini, J.C. D'Olivio, and R. Montemayer, Phys. Rev. D61, 105004 (2000).

[21] J.N. Bahcall, S. Basu, and M. Pinsonneault, 'Phys. Lett. B433, 1 (1998).

[22] A. Dressler, D. Lynden-Bell, et al., Astrophys. J. 313. 42 (1987).

[23] G.L. Fogli and E. Lisi, Astropart. Phys. 3 (1995) 185.

[24] http://www.sns.ias.edu/ jnb/SNdata.

[25] J.N. Bahcall, E. Lisi, and P.I. Krastev Phys. Rev. C55, 494 (1997).

[26] M. Smy, (The Super-Kamiokande collaboration), hep-ex/9903034.

[27] For a discussion on the sources of the various errors see the second reference of [2].

[28] J.N. Bahcall et al., Phys. Rev. D54, 411 (1996).

[29] Sudbury Neutrino Observatory Proposal, Report No. SNO-87-12 (1987).

[30] The cross-sections are calculated using the routine Deuteron.cross available at [24]. We use the charged and neutral current cross-sections obtained following K. Kubodera and S. Nozawa, Int. J. Mod. Phys. E3, 101 (1994).

[31] Y. Suzuki (private communication).

[32] J. Pantaleone, T.K. Kuo, and S.W. Mansour, Phys. Rev. D61, 033011 (2000). 


\section{Figure Captions}

Fig. 1. The $90 \%$ C.L. allowed region in the $\sin ^{2} 2 \theta-\Delta F$ plane from an analysis of the total rates seen by the Chlorine, Gallex, SAGE, Kamiokande, and SuperKamiokande (1117-day data) detectors assuming conversion due to VEP to (a) active neutrinos and (b) sterile neutrinos. The best fit points have been indicated.

Fig. 2. The region bounded by the solid lines is the $90 \%$ C.L. allowed region in the $\sin ^{2} 2 \theta$ - $\Delta F$ plane from the fitting of the 1117-day SK recoil electron spectrum data with the SSM flux normalizations $\left(X_{B}=X_{h}=1\right)$. The best fit point is marked with a dark dot. The best-fit point for the case $X_{h}=1, X_{B}$ arbitrary, is indicated by a ' $\times$ ' sign and the corresponding $90 \%$ C.L. disallowed region is enclosed by the broken lines. The two panels correspond to (a) active neutrinos and (b) sterile neutrinos.

Fig. 3. The $90 \%$ C.L. allowed region in the $\sin ^{2} 2 \theta-\Delta F$ plane from the combined analysis of total rates and 1117-day SK recoil electron spectrum data for conversion due to VEP. The hep flux normalization is held fixed at the SSM value $\left(X_{h}=1\right)$ but the ${ }^{8} \mathrm{~B}$ flux normalization $\left(X_{B}\right)$ is allowed to vary. The best fit points are indicated. Panel (a) represents the case of conversion to an active neutrino while panel (b) corresponds to conversion to a sterile neutrino.

Fig. 4. The number of events expected per day at SNO due to (a) $\nu-e$ scattering, and (b) the charged current $\nu_{e}-d$ reaction. Here $E_{e}$ stands for the observed electron energy. Results are shown for the best-fit values of the parameters obtained from fits to the spectrum and jointly to the rates and the spectrum (see text for more details). In both panels the solid (large dashed) line corresponds to the best-fit parameters obtained from a fit to the electron energy spectrum for VEP oscillation to an active (sterile) neutrino. The dot-dashed (dotted) lines similarly correspond to the best combined fits to the rates and spectrum for VEP oscillations to an active (sterile) neutrino. The BP98 SSM expectations are the small dashed curves.

Fig. 5. The ratio of the predicted number of events from VEP to the SSM expectation as a function of the electron energy for SNO due to (a) $\nu-e$ scattering, and (b) the charged current $\nu_{e}-d$ reaction (see text for more details). Here $E_{e}$ stands for the observed electron energy. In both panels the solid (large dashed) line corresponds to the best-fit parameters obtained from a fit to the electron energy spectrum for VEP oscillation to an active (sterile) neutrino. The dot-dashed (dotted) lines similarly correspond to the best combined fits to the rates and spectrum for VEP oscillations to an active (sterile) neutrino. 


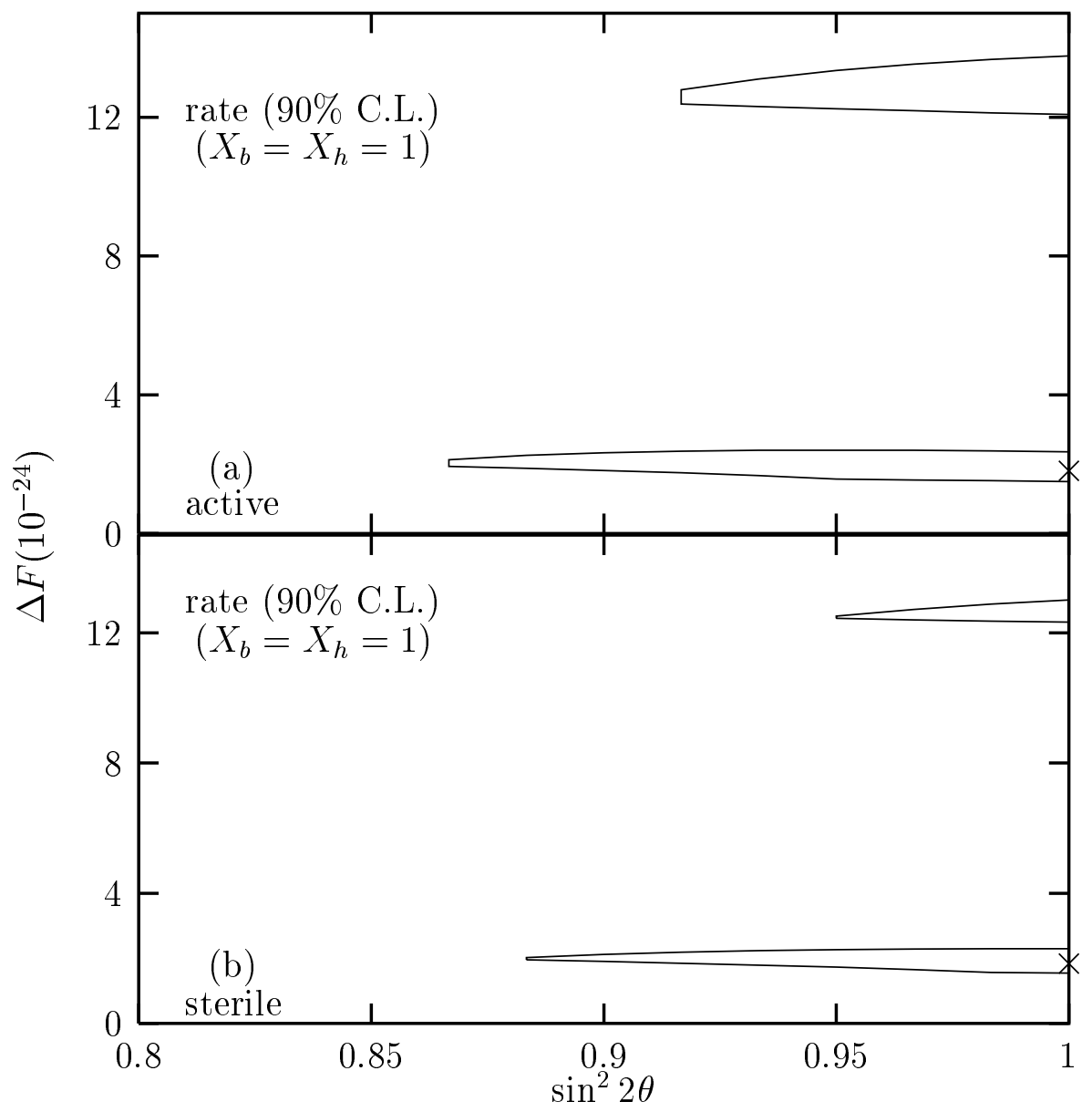

Fig.1 


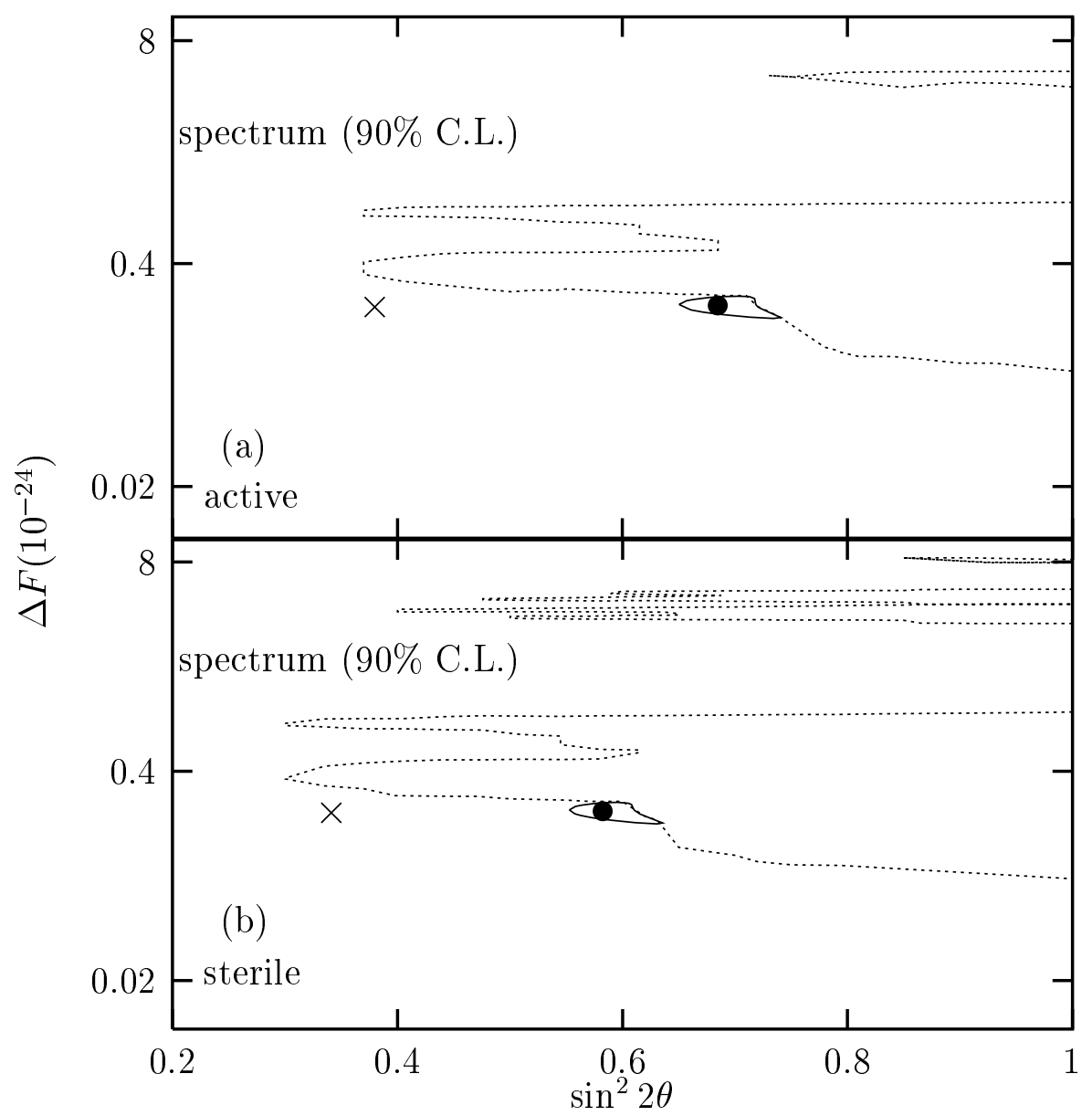

Fig.2 


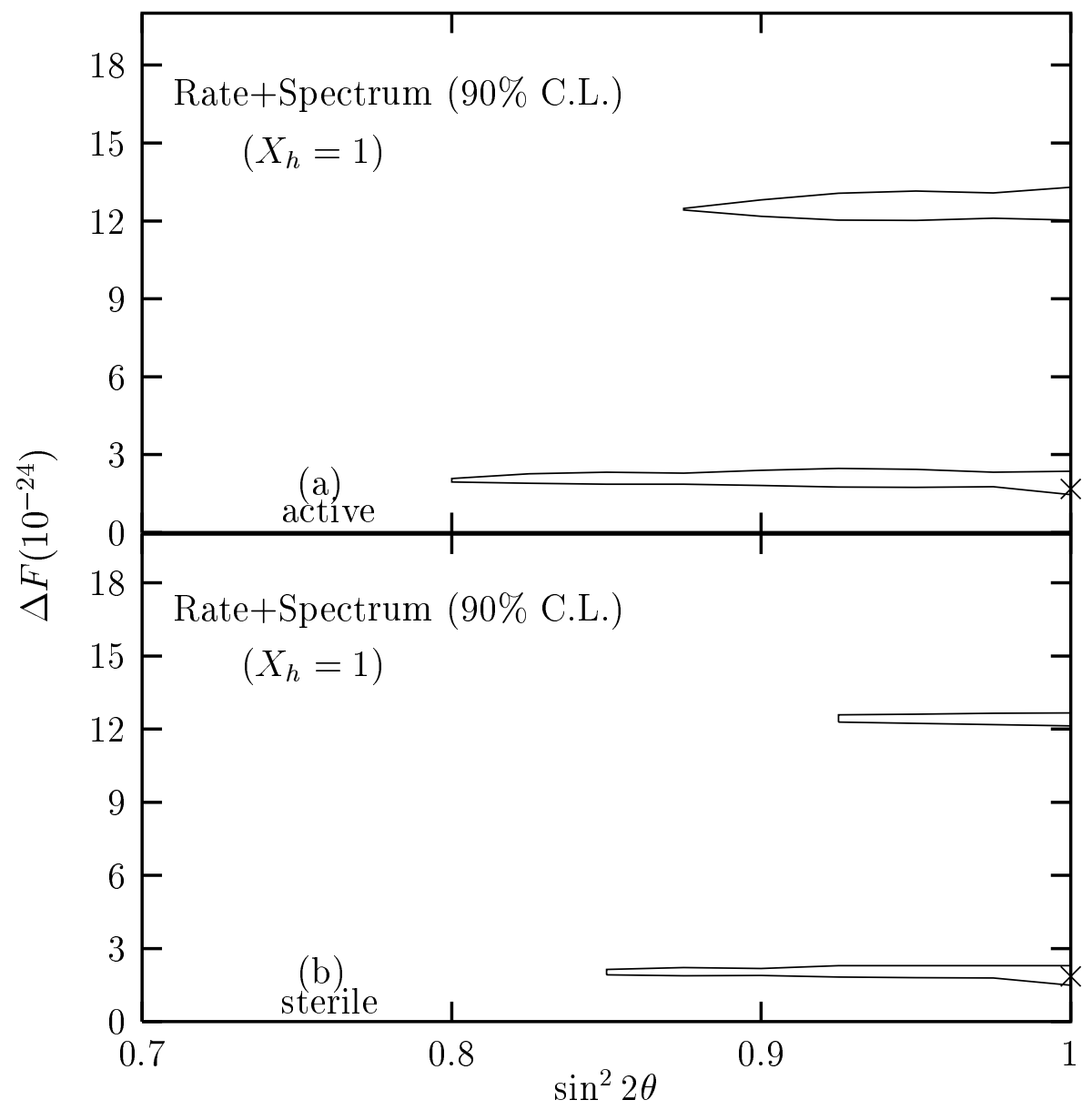

Fig.3 


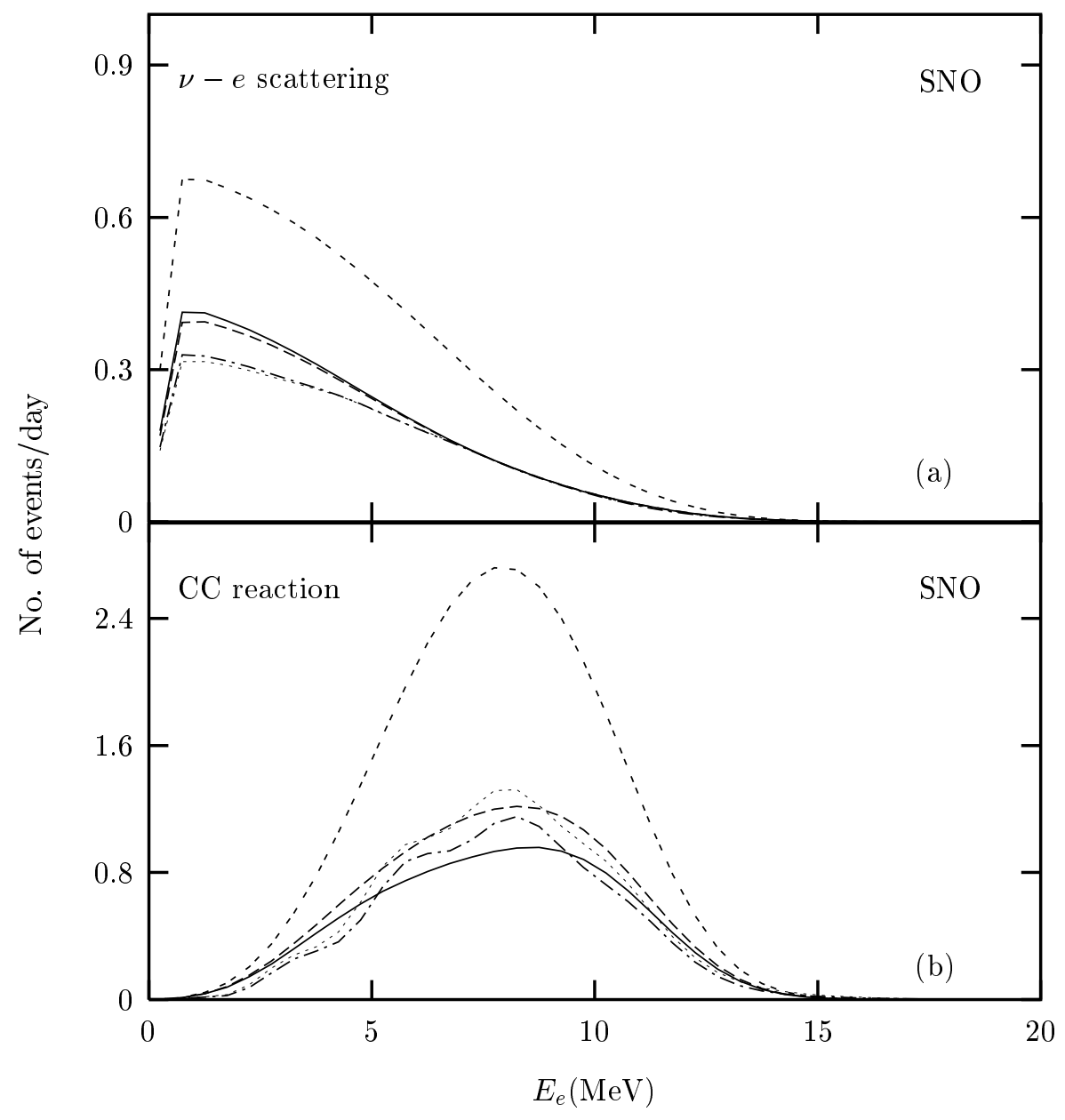

Fig.4 


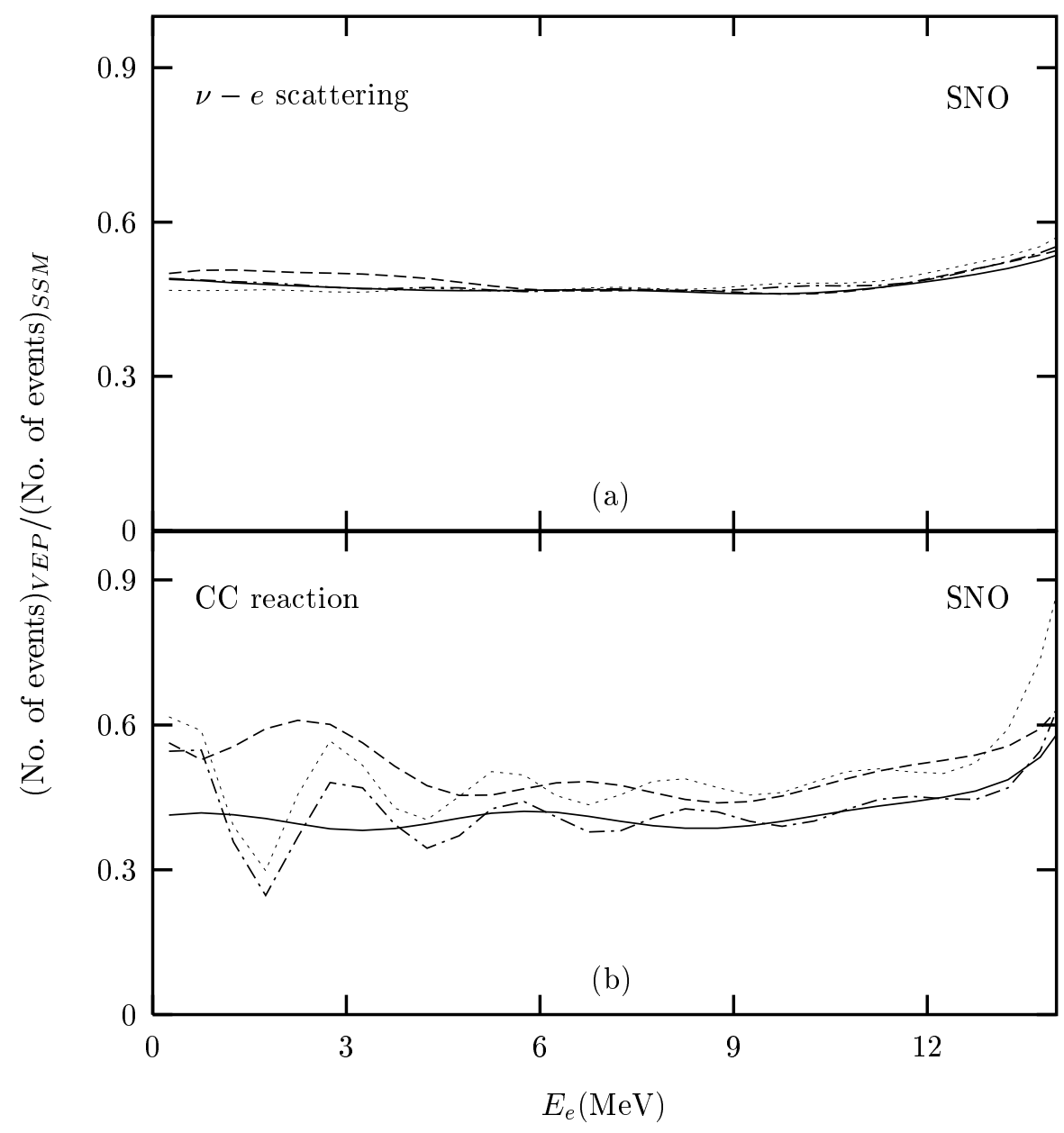

Fig.5 